\title{
Une nouvelle ère pour la génétique du rat
}

> Le rat et la souris sont, depuis déjà longtemps, des modèles de choix pour l'étude de la biologie des mammifères : faciles d'entretien, ils offrent aussi toute une panoplie de lignées génétiquement pures et de mutants bien caractérisés. Bien que longtemps préféré à la souris en raison de sa taille et de sa physiologie, le rat a progressivement été délaissé au profit de la souris, espèce chez laquelle les techniques de mutagenèse dirigée alimentent depuis plus de vingt ans le répertoire des modèles d'études. Aujourd'hui, deux avancées techniques devraient changer la donne: l'utilisation de nucléases artificielles à doigts de zinc pour inactiver un gène d'intérêt dès les premiers stades du développement et la dérivation et la manipulation génétique de cellules ES (embryonic stem cells) de rat. Nul doute que les années qui viennent verront se multiplier les modèles de maladies humaines établies chez le rat. <

Élevés de longue date comme animaux de compagnie, rats et souris se sont imposés comme modèles de choix pour l'étude de la biologie des mammifères. Outre leur petite taille, leur facilité d'entretien en laboratoire (très peu d'exigences nutritionnelles) et leur prolificité légendaire, il existe dans ces espèces des lignées pures (consanguines) caractérisées génétiquement et une très grande collection de mutants, deux ressources abondamment exploitées par les scientifiques. Séparées par l'évolution depuis 13 à 15 millions d'années, ces deux espèces de rongeur présentent quelques différences. En biologie expérimentale, un avantage certain du rat sur la souris tient à sa plus grande taille facilitant certaines manipulations et interventions chirurgicales. Les capacités cognitives sont plus développées chez le rat qui a logiquement été adopté comme modèle dans de nombreuses études de comportement et d'apprentissage. Le rat est également plus utilisé que la souris comme modèle pour des pathologies cardiovasculaires

\section{Michel Cohen-Tannoudji, Jean-Louis Guénet}

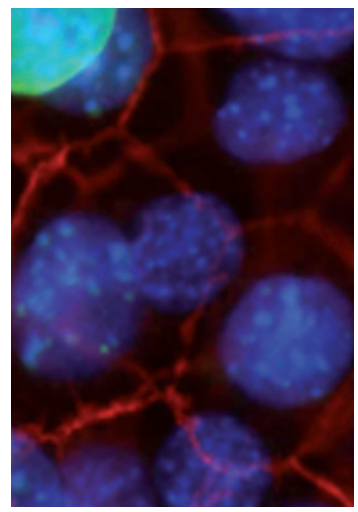

Unité de génétique fonctionnelle de la souris, CNRS URA 2578, Institut Pasteur, 25 , rue du Docteur Roux, 75724 Paris Cedex 15, France. m-cohen@pasteur.fr

telles que l'hypertension ou les accidents vasculaires cérébraux. Avec d'autres, ces différences expliquent pourquoi le rat a été pendant longtemps préféré à la souris dans plusieurs domaines de la biologie comme la physiologie, la pharmacologie et la neurobiologie [1].

À la fin du siècle dernier, l'avènement des techniques de mutagenèse dirigée par recombinaison homologue dans les cellules ES (embryonic stem) $[2,3]$ a radicalement changé la donne. L'éventail et la sophistication des modifications génétiques réalisables chez la souris ont fait de cette espèce un modèle incontournable dans la quasi-totalité des domaines d'étude de la biologie des mammifères. Si le développement de protocoles optimisés de mutagenèse aléatoire, tels que la mutagenèse chimique avec la $\mathrm{N}$-éthyl-N-nitroso-urée (ENU) [4] ou la mutagenèse insertionnelle suite à la mobilisation de transposons [5], a permis d'obtenir des rats mutants, la communauté scientifique attendait, depuis de nombreuses années, la mise au point de technologies de mutagenèse dirigée utilisables dans cette espèce. C'est maintenant chose faite grâce à deux avancées majeures récentes.

\section{Couper pour mieux inactiver}

Utilisation de nucléases artificielles: le principe

Toute cassure double brin de I'ADN doit être réparée pour maintenir l'intégrité de l'information génétique et la survie de la cellule. Chez les mammifères, la majorité des cassures sont réparées par le mécanisme dit de non homologous end joining (NHEJ). Celui-ci, peu fidèle, restaure la continuité du chromosome mais s'accompagne fréquemment de l'addition ou de la perte de quelques nucléotides pouvant, le cas échéant, avoir des conséquences fonctionnelles sévères. Une nouvelle technologie de ciblage de gènes, basée sur l'induction in embryo d'une cassure double brin ciblée au niveau d'un gène d'intérêt et la réparation approximative de celle-ci, a récemment vu le jour. Elle repose sur l'utilisation 
de nucléases artificielles comprenant, d'une part, des doigts de zinc permettant la fixation à une séquence d'ADN préalablement sélectionnée, et d'autre part le domaine nucléase de l'enzyme de restriction Fokl. Inactif à l'état de monomère, ce domaine est capable, après dimérisation, de couper n'importe quelle séquence d'ADN. L'utilisation de deux nucléases comportant des doigts de zinc judicieusement choisis pour lier spécifiquement des séquences d'ADN contiguës permet la dimérisation du domaine Fokl et l'induction d'une cassure double brin bien localisée (Figure 1). L'inactivation de gènes par cette technique a été obtenue dans de nombreuses espèces comme le maïs, le tabac, l'arabette des dames, le nématode, la drosophile ou le poisson-zèbre. Chez les mammifères, c'est le rat qui a ouvert la voie. En effet, il a été montré dans cette espèce que la simple micro-injection dans le zygote d'ARN codant pour des nucléases à doigts de zinc spécifiques permettait d'obtenir des mutations dans un gène ciblé à une fréquence très élevée [6]. Dans cette première étude, $17 \%$ des ratons nés à la suite de cette manipulation étaient porteurs d'altérations génétiques au niveau de sites de coupures situés dans le gène lgM, le gène Rab38 ou un transgène préalablement intégré dans le génome par transgenèse classique. Quelques mois plus tard, une deuxième étude confirmait ces résultats en ciblant le gène codant la chaîne $\gamma$ du récepteur de l'interleukine 2, et permettait l'obtention de rats présentant un déficit immunitaire combiné sévère et devenus tolérants à la greffe de cellules d'origine humaine [7].

\section{Inactivation génétique par nucléases artificielles: beaucoup d'avantages}

Cette technique d'inactivation de gènes est séduisante pour plusieurs raisons. D'abord, parce qu'une fois les nucléases spécifiques obtenues, la technique permet de produire rapidement des animaux mutants. Des mutations de type «perte de fonction » sont obtenues avec une efficacité tellement élevée que quelques séances de micro-injection suffisent pour obtenir des rats mutants. L'efficacité est telle que parfois les deux allèles du gène ciblé sont simultanément modifiés dans les animaux fondateurs $[6,8]$. II faut néanmoins garder à l'esprit que des modifications peuvent encore survenir plusieurs jours après l'injection et se retrouver ainsi à l'état mosaïque chez les animaux fondateurs $[7,8]$. Un deuxième atout de cette technique est qu'elle est applicable à toutes sortes de zygotes sous réserve que ceux-ci supportent la micro-injection. Ainsi, il est possible de produire des mutations dans toutes sortes de fonds génétiques et les deux premières études ont fait appel aussi bien à des lignées de rats consanguins (SS, FHH, F344/Stm, TM/Kyo) que non consanguins (Sprague Dawley). Comme attendu, cette approche est aussi en train de se propager rapidement à d'autres espèces de mammifères. Appliquée à la souris dans deux fonds génétiques différents (C57BL/6 et FVB), elle donne des résultats tout à fait comparables à ceux obtenus chez le rat $[8,9]$, et un premier modèle de lapin chez lequel les deux allèles du gène CFTR (cystic fibrosis transmembrane conductance regulator, dont les mutations sont impliquées dans la mucoviscidose) ont été inactivés a tout récemment été obtenu par cette approche (L. Boulanger, J.P. Renard, communication personnelle). Un troisième avantage de cette technique est qu'elle permet de produire une grande variété de mutations (délétions de quelques pb ou de plus d'un kb, plus rarement insertions de quelques $\mathrm{pb}$ ) qui constituent autant de sources d'informations potentielles sur la fonction du gène étudié. II faut également noter que l'absence d'introduction d'ADN exogène dans le génome devrait s'accompagner d'une meilleure acceptation par le grand public des organismes génétiquement modifiés obtenus par cette approche.

Contre toute attente, l'expression de nucléases artificielles dans les cellules embryonnaires aux premiers stades de développement n'est pas toxique et semble avoir une activité de cassure de l'ADN limitée à la séquence choisie par l'expérimentateur. En effet, l'examen des régions du génome qui présentent une séquence proche de celle reconnue par les nucléases à doigts de zinc utilisées n'a révélé jusqu'à présent aucune mutation [6-8]. De toute façon, dans l'hypothèse où de telles modifications non désirées surviendraient, elles seraient dans la plupart des cas rapidement perdues au cours des croisements nécessaires à l'établissement des lignées mutantes. Actuellement, la limite principale de cette technique réside dans la disponibilité et le coût des nucléases à doigts de zinc. Les travaux réalisés jusqu'à présent chez le rat et la souris ont tous fait appel à une même source commerciale mais il est probable qu'à l'avenir d'autres sources, y compris académiques, verront le jour.

Enfin, un développement essentiel de cette technique réside dans l'introduction in ovo de modifications plus variées (knock-in, mutations conditionnelles, etc.) dans le gène ciblé. On sait depuis longtemps que, dans des systèmes cellulaires, l'induction ciblée d'une cassure double brin stimule localement les processus endogènes de réparation du génome et permet d'augmenter considérablement la fréquence de recombinaison homologue (voir par exemple [10-13]). La faisabilité de cette stratégie a récemment été démontrée chez la souris en co-injectant dans des zygotes des ARN codant pour des nucléases à doigts de zinc et une matrice de réparation présentant des séquences d'homologies de part et d'autre de la cassure. Cette manipulation a permis l'introduction par recombinaison homologue dans le locus Rosa26 des gènes rapporteurs lacZ et Venus avec une fréquence raisonnable ( $0,3 \%$ des zygotes réimplantés $/ 2,5 \%$ des embryons en fin de gestation) [9]. D’autres études seront nécessaires pour évaluer l'efficacité de cette approche sur d'autres locus et dans d'autres espèces de mammifères.

\section{Des cellules pluripotentes de rat... enfin !}

L'obtention de cellules souches embryonnaires à partir d'embryons de souris a été rapportée au début des années 1980 [14, 15] et a valu le Prix Nobel de médecine à leurs auteurs [2]. Dès lors, de nombreuses équipes ont 

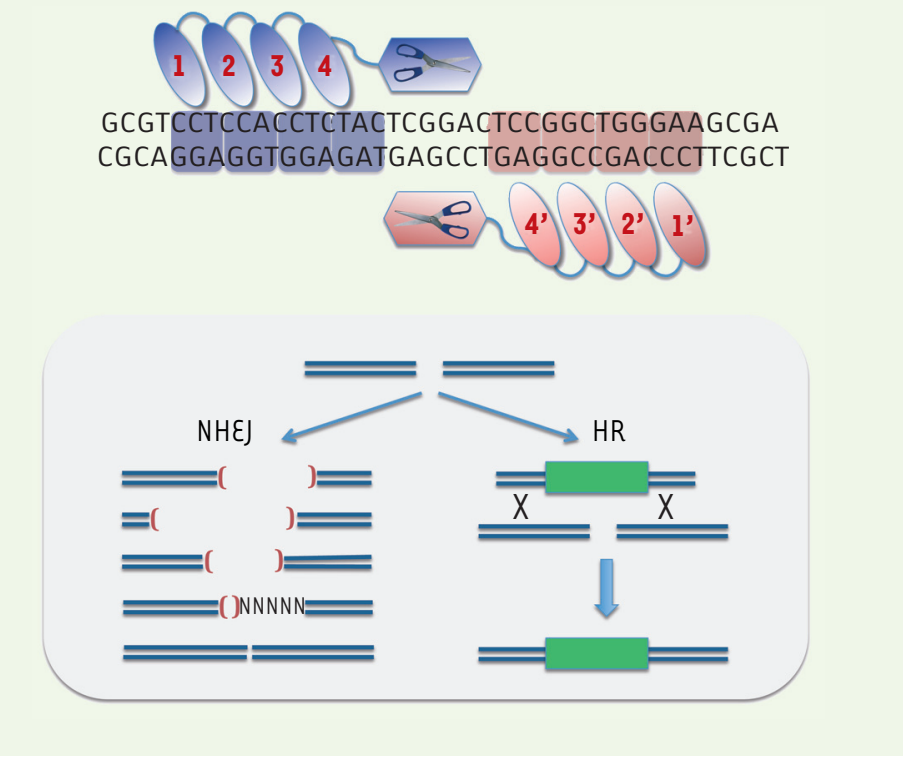

Figure 1. Principe du ciblage de gènes à l'aide de nucléases à doigts de zinc. Haut : Les nucléases à doigts de zinc sont des protéines artificielles comportant un domaine de liaison à l'ADN et un domaine de clivage (ciseaux). La fixation à I'ADN est assurée par plusieurs motifs en doigt de zinc (1, l' à 4, 4'), liant chacun trois nucléotides. Les combinatoires de doigts de zincs sont choisies de telle sorte que les deux nucléases lient des régions d'ADN rapprochées permettant ainsi la dimérisation et l'activation du domaine de clivage. Une coupure double brin de l'ADN peut ainsi être induite localement dans une région prédéfinie du génome. Bas : La cassure double brin de I'ADN peut alors être réparée selon deux voies. La non homologous end joining (NHEJ), mécanisme sujet à l'erreur, s'accompagne fréquemment de délétions et d'insertions pouvant provoquer des mutations perte de fonction. La cassure peut également être réparée par recombinaison homologue $(\mathrm{RH})$ en prenant comme modèle une matrice de réparation présentant des séquences d'homologies de part et d'autre de la cassure. Les modifications présentes sur cette matrice sont alors recopiées à l'identique sur le chromosome.

tenté sans succès d'isoler des cellules de rat ayant les mêmes propriétés, c'est-à-dire celles de cellules embryonnaires pluripotentes et possédant la capacité, après injection dans un jeune embryon, de coloniser tous les tissus, y compris sa lignée germinale. En fait, pour des raisons qui ne sont toujours pas entièrement comprises, les conditions de dérivation de cellules ES, initialement établies par Martin Evans et Gail Martin, sont particulièrement adaptées à la lignée de souris $129 \mathrm{~Sv}$, beaucoup moins voire pas du tout aux autres lignées de souris. Grâce aux travaux du laboratoire d'Austin Smith au Royaume-Uni, qui portent sur les signaux qui confèrent à une cellule le statut de cellule souche pluripotente, une nouvelle condition plus permissive de dérivation et de culture de cellules $\varepsilon S$ a été mise au point [16]. Plutôt que d'utiliser des sources peu définies de signaux favorisant le maintien à l'état indifférencié (sérum, fibroblastes embryonnaires), cette nouvelle condition de culture sans sérum communément appelée « $2 \mathrm{i}$ » fait appel à deux inhibiteurs spécifiques des kinases Mek/Erk et Gsk3 [17-19]. L'inhibition de la voie de signalisation Erk bloque efficacement la différenciation des cellules ES alors que l'inhibition de Gsk3 promeut la survie et la prolifération de celles-ci. En condition 2i, des lignées de cellules ES ont pu être aisément dérivées à partir de plusieurs lignées de souris, y compris la lignée NOD (non obese diabetic), particulièrement réfractaire jusqu'alors à la dérivation de cellules ES [19, 20]. Les nouvelles conditions de culture ont été testées et adaptées au rat, ce qui permit l'obtention de lignées de cellules ES bona fide, capables de donner des chimères germinales, à partir de trois fonds génétiques différents Dark Agouti (DA), Sprague Dawley (SD) et Brown-Norway (BN) [21-23]. Les premières lignées de cellules ES de rat, dérivées en utilisant un troisième inhibiteur, spécifique du récepteur au FGF, se sont révélées difficiles à maintenir en culture à l'état diploïde [21]. Parmi toutes les conditions testées, la culture en milieu $2 \mathrm{i}$, en présence de cellules nourricières (fibroblastes embryonnaires ou cellules L) et/ou de LIF (leukemia inhibitory factor), semble donner les résultats les plus prometteurs. Ainsi, une équipe américaine vient de démontrer qu'il est possible d'utiliser la recombinaison homologue dans les cellules $\varepsilon S$ de rat pour produire des animaux déficients pour le gène suppresseur de tumeur $p 53$ [24]. C'est une avancée considérable pour le développement de rats modèles de maladies génétiques humaines. Bien sûr, nous n'en sommes qu'au tout début et il sera important de déterminer rapidement si d'autres locus peuvent être ciblés avec la même efficacité, comme semble l'indiquer une publication très récente qui porte sur le ciblage du gène Hprt de rat [25]. La principale difficulté rencontrée lors de la génération de rats $p 53$ knock-out concernait I'instabilité chromosomique du clone de cellules ES ayant subi l'événement de recombinaison homologue. Les auteurs ont été contraints de sous-cloner le clone original afin d'isoler des cellules euploïdes capables de donner des chimères germinales. L'optimisation des conditions de culture devrait faciliter la manipulation et la culture à long terme des cellules $\varepsilon S$ et plusieurs pistes restent encore à explorer. À cet égard, une condition de culture combinant sérum de veau fœtal et $4 \mathrm{i}$ (inhibiteurs spécifiques de Mek, Gsk, Rock et Alk5) a permis d'obtenir des lignées de cellules ES à partir de rats Wistar transgéniques [22]. De même, la culture dans une atmosphère ayant une tension d'oxygène réduite pourrait favoriser l'isolement et la culture de certaines lignées de cellules ES de rat [25]. Ainsi que cela a été bien établi chez la souris, le taux de chimères germinales obtenu après micro-injection de cellules \&S dans un embryon hôte au stade blastocyste semble, chez le rat aussi, dépendre du fonds génétique des deux composants de la chimère. Ainsi, la lignée de cellules $\varepsilon S$ de fonds DA participe à la 
lignée germinale lorsqu'elle est injectée dans des blastocystes Fisher 344 (F344), alors que ce n'est pas le cas avec des blastocystes de la lignée SD [23]. De nouvelles combinatoires seront testées dans le futur et permettront certainement d'améliorer l'efficacité de la procédure de mutagenèse dirigée. Enfin, il faut signaler que les nouvelles conditions de culture ont également permis d'obtenir d'autres types de cellules souches pluripotentes de rat, tels que $\varepsilon G$ (embryonal germinal) [26] et iPS (induced pluripotent stem cells) [27-29], augmentant par là même le répertoire des expériences envisageables dans cette espèce.

\section{En conclusion}

II ne fait guère de doute que de nombreux rats porteurs de modifications ciblées de leur génome verront le jour dans les années qui viennent, et la communauté scientifique doit se préparer à gérer et exploiter au mieux cette nouvelle ressource. On ne peut que se réjouir de la multiplicité des modèles. L'étude comparée d'une même altération génétique dans plusieurs espèces permet non seulement de consolider les conclusions tirées mais également de pointer les particularités propres à chaque espèce et d'identifier les innovations biologiques survenues au cours de l'évolution. Enfin, au-delà du rat, la voie est ouverte pour manipuler de façon contrôlée le génome de mammifères de plus grande taille et produire notamment de meilleurs modèles homologues de maladies génétiques humaines complémentaires aux modèles rongeurs. $\diamond$

\section{SUMMARY}

\section{A new era in rat genetics}

Rat and mice are privileged tools for scientists. However, despite obvious advantages, such as a larger size, more faithful reproduction of human diseases, and utility for physiological and cognitive studies, rats have suffered from limited genetic technologies such as targeted mutagenesis. However, the gap between rat and mouse for genetic approaches will soon disappear with the recent advances of zinc finger nucleases applicable to early-stage rat embryos and the successful derivation of germ line competent rat ES cells, almost thirty years after murine $\varepsilon S$ cells. This will lead to new opportunities and to increase our capacity to model human pathologies. $\diamond$

\section{CONFLIT D'INTÉRÊTS}

Les auteurs déclarent n'avoir aucun conflit d'intérêts concernant les données publiées dans cet article.

\section{RéFÉRENCES}

1. Aitman TJ, Critser JK, Cuppen $\varepsilon$, et al. Progress and prospects in rat genetics: a community view. Nat Genet 2008 ; $40:$ : 516-22.

2. Cohen-Tannoudji M. Prix Nobel de médecine 2007. Des souris mutantes à façon. Med Sci (Paris) $2007 ; 23: 1159-61$.

3. Babinet C, Cohen-Tannoudji M. Vingt ans d'interventions délibérées sur le génome de la souris. Une révolution dans l'approche génétique de la biologie des mammifères. Med Sci (Paris) 2000 ; $16: 31-42$.

4. Mashimo T, Yanagihara K, Tokuda S, et al. An ENU-induced mutant archive for gene targeting in rats. Nat Genet $2008 ; 40: 514-5$.

5. Izsvak Z, Frohlich J, Grabundzija I, et al. Generating knockout rats by transposon mutagenesis in spermatogonial stem cells. Nat Methods $2010 ; 7: 443-5$.
6. Geurts AM, Cost GJ, Freyvert Y, et al. Knockout rats via embryo microinjection of zinc-finger nucleases. Science $2009 ; 325: 433$.

7. Mashimo T, Takizawa A, Voigt B, et al. Generation of knockout rats with $X$-linked severe combined immunodeficiency $(X-S C I D)$ using zinc-finger nucleases. PLoS One $2010 ; 5$ : e8870.

8. Carbery ID, Ji D, Harrington A, et al. Targeted genome modification in mice using zinc-finger nucleases. Genetics $2010 ; 186: 451-9$.

9. Meyer M, de Angelis MH, Wurst W, Kuhn R. Gene targeting by homologous recombination in mouse zygotes mediated by zinc-finger nucleases. Proc Natl Acad Sci USA 2010 ; 107 : 15022-6.

10. Cohen-Tannoudji M, Robine S, Choulika A, et al. I-Scel-induced gene replacement at a natural locus in embryonic stem cells. Mol Cell Biol 1998 ; $18: 1444-8$.

11. Donoho G, Jasin M, Berg P. Analysis of gene targeting and intrachromosomal homologous recombination stimulated by genomic double-strand breaks in mouse embryonic stem cells. Mol Cell Biol $1998 ; 18: 4070-8$.

12. Porteus MH, Carroll D. Gene targeting using zinc finger nucleases. Nat Biotechnol $2005 ; 23: 967-73$.

13. Zou J, Maeder ML, Mali P, et al. Gene targeting of a disease-related gene in human induced pluripotent stem and embryonic stem cells. Cell Stem Cell $2009 ; 5: 97-110$.

14. Evans MJ, Kaufman MH. Establishment in culture of pluripotential cells from mouse embryos. Nature $1981 ; 292$ : 154-6.

15. Martin GR. Isolation of a pluripotent cell line from early mouse embryos cultured in medium conditioned by teratocarcinoma stem cells. Proc Natl Acad Sci USA $1981 ; 78: 7634-8$.

16. De Vos J. Cellules souches pluripotentes de rat: so what? Med Sci (Paris) $2009 ; 25: 447-8$.

17. Nichols J, Smith A. The origin and identity of embryonic stem cells. Development $2011 ; 138: 3-8$.

18. Silva J, Barrandon 0, Nichols J, et al. Promotion of reprogramming to ground state pluripotency by signal inhibition. PLoS Biol $2008 ; 6$ : e253.

19. Ying QL, Wray J, Nichols J, et al. The ground state of embryonic stem cell self-renewal. Nature $2008 ; 453: 519-23$.

20. Nichols J, Jones K, Phillips JM, et al. Validated germline-competent embryonic stem cell lines from nonobese diabetic mice. Nat Med 2009; 15 : 814-8.

21. Buehr M, Meek S, Blair K, et al. Capture of authentic embryonic stem cells from rat blastocysts. Cell $2008 ; 135: 1287-98$.

22. Kawamata M, Ochiya T. Generation of genetically modified rats from embryonic stem cells. Proc Natl Acad Sci USA 2010 ; 107 : 14223-8.

23. Li P, Tong C, Mehrian-Shai R, et al. Germline competent embryonic stem cells derived from rat blastocysts. Cell $2008 ; 135$ : 1299-310.

24. Tong C, Li P, Wu NL, et al. Production of $\mathrm{p} 53$ gene knockout rats by homologous recombination in embryonic stem cells. Nature $2010 ; 467: 211-$ 3.

25. Meek S, Buehr M, Sutherland L, et al. Efficient gene targeting by homologous recombination in rat embryonic stem cells. PLoS One 2010; 5 : el4225.

26. Leitch HG, Blair K, Mansfield W, et al. Embryonic germ cells from mice and rats exhibit properties consistent with a generic pluripotent ground state. Development 2010 ; 137 : 2279-87.

27. Kobayashi T, Yamaguchi T, Hamanaka S, et al. Generation of rat pancreas in mouse by interspecific blastocyst injection of pluripotent stem cells. Cell $2010 ; 142: 787-99$.

28. Liao J, Cui C, Chen S, et al. Generation of induced pluripotent stem cell lines from adult rat cells. Cell Stem Cell $2009 ; 4: 11-5$.

29. Li W, Wei W, Zhu S, et al. Generation of rat and human induced pluripotent stem cells by combining genetic reprogramming and chemical inhibitors. Cell Stem Cell 2009; 4 : 16-9.
TIRÉS À PART

M. Cohen-Tannoudji 